\title{
Optimizing hydrogen transportation system for mobility via compressed hydrogen trucks
}

\author{
Amin Lahnaoui ${ }^{1)}$, Christina Wulf ${ }^{1)}$, Heidi Heinrichs ${ }^{1)}$, Didier Dalmazzone ${ }^{2)}$ \\ 1) Forschungszentrum Jülich, Institute of Energy and Climate Research - Systems Analysis and Technology \\ Evaluation (IEK-STE), D-52425 Jülich, Germany \\ 2) ENSTA ParisTech, 828, boulevard des Maréchaux, 91120 PALAISEAU, France
}

Contribution to The $7^{\text {th }}$ world hydrogen technology convention - WHTC2017 + IJHE

\section{Executive Summary}

Defining and installing an adequate cost-effective infrastructure for hydrogen mobility is still the main barrier restraining its deployment. This study aims to provide the minimum cost related to deploying hydrogen infrastructure based on the use of compressed gas trucks (CGT).

First, as the current market offers a variety of CGT, four pressure levels are taken into account in the optimization problem. The levelized cost of transporting hydrogen (LCOTH) associated with each one is formulated then, as a function of the transported capacity and distance. This cost function includes the costs related to the compression, storage and road transportation.

LCOTH is then minimized by optimizing the capacities transported by each CGT. The results showed that $L C O T H$ decreases with the transported capacity and increases with the trip distance. The main used CGT are the ones at high pressure (above 500 bar). Their share increases with the distance and the hydrogen demand.

\section{Keywords}

Hydrogen infrastructure, transportation, storage, compression, cost optimization, compressed gas truck 


\section{Nomenclature}

$\begin{array}{ll}C G T & \text { Compressed gas truck } \\ \text { FCEV } & \text { Fuel cell electric vehicle } \\ T P D & \text { Tons per day } \\ i, j & \text { Locations } \\ S^{0}, S^{t} & \text { Hydrogen state at atmospheric pressure } P_{0} \text { and operating pressure } P_{t} \text { respectively } \\ t & \text { Operated by a CGT where the hydrogen is at pressure level of } P_{t} \\ C & \text { Associated to compression cost } \\ S & \text { Associated to storage cost } \\ T & \text { Associated to transportation cost } \\ t u b e & \text { Associated to the tube cost } \\ \text { und } & \text { Associated to the undercarriage cost } \\ \text { Tra } & \text { Associated to the trailer cost (both the tube and the undercarriage costs) } \\ \text { Cab } & \text { Associated to cab cost }\end{array}$

\section{Technical parameters}

$d_{i j}$

$\Delta d$

$C p$

$\triangle C p$

$\dot{W}_{\Delta S \rightarrow 0}$

$\dot{W}_{m}$

$\gamma$

$N$

$\eta_{c}$

$P^{t}$

$P_{0}$

$T_{0}$

$P_{b, C}$

$S_{b, C}$

$m$

$\Delta m$

$P_{b, S}$

$S_{b, S}$
Distance in $\mathrm{km}$ between the locations $i$ and $j$

Distance step in km

Total hydrogen capacity transported in $\mathrm{kg}$

Hydrogen capacity step in $\mathrm{kg}$

Adiabatic work in $\mathrm{kWh} / \mathrm{kg}$

Compressor electrical work in $\mathrm{kWh} / \mathrm{kg}$

Specific heat ratio of hydrogen

Compressor stages

Compressor electrical efficiency

Operating pressure in bar

Atmospheric pressure in bar

Ambient temperature in $\mathrm{K}$

Baseline operating pressure for compression in bar

Base compressor size in $\mathrm{kg}$

Total transported capacity by one CGT in kg

One CGT capacity step in $\mathrm{kg}$

Baseline operating pressure for storage in bar

Base tank capacity in $\mathrm{kg}$ 


$\begin{array}{ll}t t_{l / u} & \text { Total loading }(\mathrm{I}) \text { and unloading }(\mathrm{u}) \text { time in hours } \\ t t_{l / u}^{\max } & \text { The maximum total loading and unloading time in hours } \\ A v . & \text { Annual availability of the truck in hours } \\ T h_{a} & \text { Total annual hours } \\ S_{a} & \text { Average truck speed in } \mathrm{km} / \mathrm{h} \\ n_{r . t} & \text { Maximum number of round trips } \\ n_{T} & \text { Number of CGT } \\ n_{d r i v e r} & \text { Number of drivers } \\ n_{w . h} & \text { Number of driver working hours }\end{array}$

\section{Cost parameters}

$\begin{array}{ll}N P V & \text { Net present value in } € \\ y n & \text { Economical life time in years } \\ i_{d r} & \text { Discount rate } \\ C_{a n} & \text { Total annual cost in } € \\ C C_{\times} & \text {Capital cost related to } \times \text { in } € \\ C R F_{\times} & \text {Capital recovery factor related to } \times \\ C F_{\times} & \text {Capacity factor related to } \times \\ O \& M_{\times} & \text {Operations and maintenance cost related to } \times \text { in } € \\ O M_{\times} & \text {O\&M } \\ F C & \text { Fuel cost in } € \\ L C & \text { Labour cost in } € \\ L C O T H & \text { Total levelized cost of transporting hydrogen in } € / \mathrm{kg} \\ L C O H_{\times} & \text {Levelized cost of hydrogen related to } \times \text { in } € / \mathrm{kg} \\ C e & \text { Unit electrical energy cost in } € 2015 \\ T C e & \text { Annual cost of energy to operate the compressor in } € \\ C_{b, C} & \text { Unit fuel cost in } € 2015 \\ F_{p} & \text { Driver wage in } € \\ T C_{d r i v e r} & \\ & \end{array}$

\section{Mathematical parameters}

$\begin{array}{lll}\lfloor\rfloor & \text { floor function defined by: } & \\ \chi(\text { ) } & \text { Step function defined by: } & \\ & \chi_{A}(x)=\left\{\begin{array}{c}1 \text { if } x \in A \\ 0 \text { if } \text { not }\end{array}\right.\end{array}$




$$
\begin{aligned}
& \min \sum L C O T H * C p^{t} \quad \text { Linear program formulated where } C p^{t} \text { represents the vector variables to determine } \\
& \text { minimize } L C O T H * C p^{t} \\
& \text { subject to } C p=\sum_{t} C p^{t} \\
& \text { and } C p^{t} \geq 0 \\
& \min \sum L C O T H * i^{t} \quad \text { Equivalent Integer linear program formulated where } i^{t} \text { represents the integer vector } \\
& \text { variables to determine } \\
& \text { minimize } L C O T H * i^{t} \\
& \text { subject to } n=\sum_{t} i^{t} \\
& \text { and } i^{t} \in \mathbb{Z}^{t} \\
& i^{t} \geq 0
\end{aligned}
$$

\section{Introduction}

One of the big challenges of the future of energy is to find a balance between the increasing demand on energy, the limited fossil fuel resources and the necessity to lower the carbon emissions. This challenge is mainly apparent in the transportation sector. On one hand, this sector has a high energy demand and its share of total energy need is still increasing. In the case of countries of the European Union, it represented 32 \% of the final energy demand consumed in 2014 [Commission, 2015]. On the other hand, the growing transportation demand increases the dependency on conventional fuel and increases carbon emissions as well. In fact, while total emissions of the European countries have decreased by $17 \%$ in the last 25 years, the transportation sector has been the only one that had its emissions increased by $22 \%$ [Commission, 2015] during the same period. To deal with that, the European Union fixed the threshold of oil dependency in transportation to be reduced by $70 \%$ in 2050 compared to its level in 2008 in order to decarbonise the transportation sector [White Paper, 2011].

The use of low carbon Fuel Cell Electric Vehicles (FCEV) is one of the promising alternatives for the conventional fuel. However, defining and installing an adequate cost-effective infrastructure is still the main barrier restraining its deployment. Many solutions for hydrogen supply chains are likely to emerge depending on level of demand, resource availability, geographic factors and progress on hydrogen technologies. Currently, the main improvement comes from the research field, which improves economic feasibility by reducing the total cost of supply or by acting on different steps of the supply chain including mainly production, storage, transportation and distribution.

Under this problematic, this study aims to provide the minimum cost for deploying hydrogen infrastructure. This is done by taking into account different hydrogen transportation modes including different trucks and hydrogen states as compressed gas, liquefied or chemically bound to a liquid carrier. First, the minimum cost of transporting hydrogen using compressed gas trucks (CGT) is calculated. The associated levelized cost of transporting hydrogen (LCOTH) includes the transportation, the compression and storage as well. 
A literature review is presented in the first part introducing the different works on hydrogen infrastructure optimization along with the reason and the methodology behind the optimal hydrogen transportation via compressed gas trucks. Then the model components of the transportation optimization are presented, which includes technical and cost evaluations of compressed storage and transportation via trucks. Finally, the mathematical model will be presented along with the main results for different hydrogen demand and location distances.

\section{Literature review}

The literature review shows examples of optimizing the total hydrogen infrastructure by limiting the analysis on one way of hydrogen transportation (a) or by investigating a part of the hydrogen delivery pathway that includes the stages of hydrogen production, storage, transmission and distribution in separate studies (b).

Analysis (a) is done either via trucks or pipeline system [Marcoulaki et al., 2012, Baufumé et al., 2013] or by including the two transportation systems in different scenarios [Demir \& Dincer, 2017]. Often the cost effectiveness of the whole supply chain was improved by analysing only one part of the pathway (b) [Stojić et al., 2003, Yang \& Ogden, 2007, Kim \& Kuby, 2012, Bellotti et al., 2015].

For instance, [Yang \& Ogden, 2007] have developed models in order to identify the cost effective delivery mode and the results showed that the demand impacted the cost. For instance, small refuelling station with low demand can be supplied using compressed gas truck, while moderate demand can be supplied using liquid hydrogen when the pipeline system could be used for dense area that have a larger hydrogen demand.

[Stojić et al., 2003] investigate the reduction of energy needs in industrial electrolysers, while [Bellotti et al., 2015] focused on the hydrogen storage process taking in account two different alternatives. The first alternative, consists of a conventional method in which the hydrogen is stored in high pressure tanks, and the second one, used storage in hydro-methane form.

Finally, [Kim \& Kuby, 2012], developed a mixed-integer linear program that optimizes the locations of the refuelling stations. Their model considered the limited driving range of automobiles and necessary deviations likely to happen when the refuelling station network is sparse.

The different optimisations were performed by focusing on one pressure level. For instance, [Yang \& Ogden, 2007] investigate the cost of transporting hydrogen using a CGT at low operating pressure of 162 bar pressure transporting a total net capacity of $300 \mathrm{~kg}$ when [Demir \& Dincer, 2017] investigate a higher operating CGT pressure of 486 bar but only at a fixed round-trip distance of $100 \mathrm{~km}$. The middle range pressure of 200 bar was investigated as well by developing 
a model based on life cycle cost for implementing a general refuelling station siting [Sun et al., 2017] or along the expressway [He et al., 2017].

The net transported capacity was chosen either variable independent from the distance trip and ranging between 250 and $460 \mathrm{~kg}$ [He et al., 2017], or fixed to $200 \mathrm{~kg}$ per trip [Dayhim et al., 2014].

This literature review showed that optimizing the overall hydrogen supply chain and delivery chain shifted the focus away from the transportation using compressed gas. In fact, this mean of transporting hydrogen could be further optimized by investigating three parameters: the pressure level, the transported capacity and trip distance.

The first one is done by investigating a set of pressure levels instead of restraining the study on only one. In fact, the current CGT market and future prospects give a range of five potential operation pressure level ranging from 180 to 540 bar, which could be used to minimize the cost of transportation.

The two other parameters can be further investigated by setting both of them as variables and extending the data range to a transported capacity up to 100 TPD and a trip distance ranging between $1 \mathrm{~km}$ and $500 \mathrm{~km}$. While the literature reviewed investigated limited transported capacity and/ or fixed trip distance, this study gives flexibility to both parameters to investigate them simultaneously.

\section{Methodology}

The model aims to find the optimum combination of CGT at different pressure levels to transport hydrogen at the minimum cost for different trip distance and different hydrogen flow demand. For that, both production and distribution are taken as input parameters for the model based on the case of France and Germany.

Concerning the choice of CGT, potential pressure levels ranging between 180 and 540 bar associated with the current and future CGT market are considered for this analysis.

The primary result showed that less than $0.001 \%$ of trucks at 180 bar are needed for the hydrogen demand and trip distances set data. Therefore, the analysis is restrained to four pressure levels presented in Table 1, along with the total net hydrogen capacity that can be transported by each CGT.

The choice was made by investigating current operating CGT in the market, literature reviews and reports [Simbeck \& Chang, 2002a, Composites, 2006, Steward et al., 2008, Tamhankar, 2014].

As the study aims to build a scenario of hydrogen penetration by 2030 , the analyses uses as well the target set by the US DOE for the year 2020 to reach economic feasibility of a filling pressure of 520 bar [Gerboni, 2016] along with other future prospects [Hexagon, 2013, Zerhusen, 2013]. 
Table 1: Variable parameters for CGT technical assessment

\begin{tabular}{l|c|r|r|r|r}
\hline Design pressure in bar & $P_{t}$ & 250 & 350 & 500 & 540 \\
\hline Total net truck capacity in kg & $m^{t}$ & 720 & 907 & 1100 & 1350 \\
\hline
\end{tabular}

Source: [Simbeck \& Chang, 2002b, Almansoori \& Shah, 2006, Steward et al., 2008, Hexagon, 2013, Zerhusen, 2013, Tamhankar, 2014, Gerboni, 2016]

The focus of the use of hydrogen in this paper is mobility via FCEVs, the hydrogen will be produced using excess wind electricity via electrolysis.

For the case of Germany, the country has already a high share of wind energy in the electricity mix [OPSD, 2018] with high disparities. In fact, the main wind generation is located in the north, where the populated and industrial areas mostly located in the south of the country.

In contrast, France by targeting a nuclear share of $50 \%$ by 2025 [Gouvernemt, August 2017] aims to reach an installed capacity of $45 \mathrm{GW}$ onshore wind power by 2030 [Eolienne, 2017].

The location of hydrogen production is supposed to be fixed and not included as a decision variable, allowing to cover the main fuel consumption for mobility. Results showed that at the national level and in case of a high hydrogen penetration of $2.4 \%$ with FCEV on the overall passenger car market by 2030 an average hydrogen demand of 338 and 379 TPD will be needed for France and Germany, respectively [Lahnaoui et al., 2017]. Therefore, a hydrogen demand ranging between 0.01 and 100 TPD is feasible for a regional need.

The hydrogen is then transported to the hydrogen refuelling stations to refuel FCEV. In this analysis, only transportation via truck is considered to minimize the cost. First, the hydrogen is compressed and stored at the production site, then transported via gas trucks over a distance ranging between 1 and $500 \mathrm{~km}$ for regional use. In fact, France and Germany present a maximum distance north to south of respectively 962 and $853 \mathrm{~km}$ as well as a maximum distance east to west of 950and $650 \mathrm{~km}$, respectively.

The transported hydrogen is finally refuelled at stations with compressors, high pressure storage and dispensers.

For the cost calculation and because the production and consumption rates are assumed fixed, only the cost related to transporting hydrogen $\mathrm{LCOTH}$ will be minimized. These costs include compression at the production site $\mathrm{LCOH}_{C}$, storage $\mathrm{LCOH}_{S}$ and transporting the compressed gas via CGT $L C O H_{T}$ as shown in Equation 1. 


\section{Input parameters}

The hydrogen gas is initially at a known location $i$ and at an initial state $S^{0}$ where the hydrogen is a gas at ambient temperature $T_{0}$ and atmospheric pressure $P_{0}$. The hydrogen is then compressed using a compressor of five stages delivering hydrogen up to 720 bar [Jensen et al., 2007] to a state $S^{t}$, where the hydrogen is a gas at ambient temperature $T_{0}$ and pressure $P^{t}$. The compressed gas at the state $S^{t}$ is stored in different tube trailers of total capacity of $m^{t}$ (Table 1) and then loaded to be transported by a CGT to a location $j$.

Once the hydrogen is transported to the final location $j$ over a distance $d_{i j}$ from the storage site $i$, the truck is supposed to wait till it is unloaded adding a total loading and unloading time $t t_{l / u}{ }^{t}$ that depends on its hydrogen state of aggregation. Hence, over the distance $d_{i j}$, each single CGT can perform a maximum annual number of roundtrips. The tube trailer transported is supposed to be filled at its maximum capacity so that the annual number of roundtrips meat the maximum $n_{r . t}{ }^{t}$. The maximum capacity $m^{t}$ again depends on hydrogen state of aggregation of the transported gas. Finally each truck is operated by a number of driver $n_{\text {driver }}$ limited by its working hours.

\section{IV.1 Technical assessment}

\section{IV.1.1 Compression work}

A five stage ( $N=5$ ) compressor delivering hydrogen up to 720 bar is chosen as it work was find out to be close to the practical one with less energy consumption [Jensen et al., 2007]. The specific work $\dot{W}_{\Delta S \rightarrow 0}{ }^{t}$ (Equation 2) in $\mathrm{kWh} / \mathrm{kg}$ is deduced from the adiabatic work [Jensen et al., 2007] of compressing hydrogen gas of specific volume equal to $11.986 \mathrm{~m}^{3} / \mathrm{kg}$ at $20^{\circ} \mathrm{C}$ [McCarty et al., 1981].

$$
\dot{W}_{\Delta S \rightarrow 0}{ }^{t}=0.3229 * N * \frac{\gamma}{\gamma-1} * P_{0} *\left[\left(\frac{P_{t}}{P_{0}}\right)^{\frac{\gamma-1}{N * \gamma}}-1\right]
$$

For a pressure given in bar, the first term includes the unit conversion from $\mathrm{J} / \mathrm{kg}$ and Pascal multiplied by the specific volume of hydrogen and $\gamma$ is the specific heat ratio of hydrogen [McCarty et al., 1981].

The annual energy to operate a compressor, which is driven by an electric motor, is deduced then from the electrical work $\dot{W}_{m}$ taking into account the total efficiency $\eta_{c}$ as a product of its adiabatic efficiency that varies between 75 and $85 \%$ and electrical one of the order of $90 \%$ (typically a total efficiency of $70 \%$ of the adiabatic work $\dot{W}_{\Delta S \rightarrow 0}$ [Jensen et al., 2007]). 


\section{IV.1.2 Transportation via truck}

The maximum annual number of roundtrips $n_{r . t}{ }^{t}$ (Equation 3 ) between two locations $i$ and $j$ depends also on the availability of the truck, which is expressed in the number of hours when the truck is available $(A v$.) for transportation during the year, and the average truck speed $S_{a}$.

$$
n_{r . t}{ }^{t}=\left\lfloor\frac{A v .}{\frac{2 * d_{i j}}{s_{a}}+t t_{l / u^{t}}}\right\rfloor
$$

In case of the use of one type CGT to transport a total hydrogen capacity $C p$ (in $\mathrm{kg}$ ) the number of trucks needed is defined in Equation 4 by:

$n_{T}^{t}=\left\lfloor\frac{C p}{m^{t} * n_{r t} t}\right\rfloor+1$

Finally, for transporting hydrogen, each driver cannot exceed a maximum number of working hours $n_{w . h}$ defining the numbers of drivers $n_{\text {driver }}{ }^{t}$ (Equation 5 ) needed to operate a CGT over a distance $d_{i j}$ by:

$$
n_{\text {driver }}{ }^{t}=\left\lfloor\frac{\frac{d_{i j}}{S_{a}}+t t_{l / u^{t}}}{n_{w . h}}\right\rfloor+1
$$

\section{IV.2 Cost assessment}

To compare investments with different economical lives of $y n$ years at a specified discount rate $i_{d r}$, the net present value (NPV) method is used (Equation 6). Taking the notation used in the definition of $\mathrm{LCOH}$ the net present value of the initial investment can be written [André et al., 2014]:

$N P V=\sum_{y=1}^{y n} \frac{C_{a n}}{\left(1+i_{d r}\right)^{y}}=C C+\sum_{y=1}^{y n} \frac{o \& M+F C+L C}{\left(1+i_{d r}\right)^{y}}$

The sum of the power series of variable year $\boldsymbol{y}$ can be written (Equation 7) using the definition of the capital recovery factor (CRF) [Short et al., 2005].

$\sum_{y=1}^{y n} \frac{1}{\left(1+i_{d r}\right)^{y}}=\frac{1-\left(1+i_{d r}\right)^{-y n}}{i_{d r}}=\frac{1}{C R F}$

Equation 6 allows then to define the annual cost $C_{a n}$ directly from the capital cost $C C$, operations and maintenance cost $O \& M$, fuel cost $F C$ and logistic costs $L C$. And using Equation 7 to replace the power series and the fact that $O \& M$ is a percentage of the capital cost $O M$, equation 6 is equivalent to Equation 8: 
The cost of transporting hydrogen is introduced via the Levelized Cost of Hydrogen Transportation (LCOHT) reflecting the annual cost of transporting one unit $\mathrm{kg}$ of hydrogen and as a sum of the different LCOHT of the hydrogen chain including the cost of compression, storage and transportation via truck.

\section{IV.2.1 Cost of compression and storage}

The annual cost of compression (Equation 11) can be broken down into the capital cost (Equation 10) and the annual cost of energy to operate the compressor (Equation 9). The last term (Equation 9) is derived from the cost of the compressors electrical work. This is calculated using its capacity factor $C F_{C}$ defined by the percentage of the annual working hour and the unit electrical energy cost $\boldsymbol{C} \boldsymbol{e}$ in euro/ kWh.Kg:

$T C e^{t}=\dot{W}_{\Delta S \rightarrow 0}^{t} * C e * C F_{C} / \eta_{c}$

The first term (Equation 10) is calculated using a sizing factor of 0.8 to adjust from the baseline size of $4000 \mathrm{~kW}$ and cost of $1164 € / \mathrm{kW}(2015 €)$ determined by the energy and cooling water requirements [Drennen \& Rosthal, 2007].

$C C_{C}{ }^{t}=C_{b, C} * S_{b, C} *\left(\frac{\dot{W}_{\Delta S \rightarrow 0}{ }^{t} * C p}{\eta_{c} * S_{b, C} * C F_{C} * T h_{a}}\right)^{0.8} *\left(\frac{P_{t}}{P_{b, C}}\right)^{0.18}$

10

Equation 11 shows the total annual cost while Table 2 shows the definitions and the values of the different parameters used for the calculation:

$\mathrm{LCOH}_{C} * \mathrm{Cp}=\mathrm{CC}_{C}{ }^{t} *\left(\mathrm{CRF}_{C}+\mathrm{OM}_{C}\right)+\mathrm{TC} e^{t} * C p$

The same methodology used above [Drennen \& Rosthal, 2007] is applied to calculate the capital cost of storing hydrogen (Equation 12) using a base case size corresponding to the one at high pressure of 540 bar at $2444 € / \mathrm{kg}(2015 €)$, this corresponds approximatively to $1252.5 \mathrm{~kg}$ net hydrogen stored.

$\mathrm{LCOH}_{S} * C p=C C_{S}{ }^{t} *\left(C R F_{S}+\mathrm{OM}_{S}\right)=C_{b, S} * S_{b, S} *\left(\frac{C p^{\frac{P_{t}}{P_{b} S}}}{S_{b, S}}\right)^{0.75} *\left(\frac{P_{t}}{P_{b, S}}\right)^{0.44}$

Table 2: Parameters for compression and storage capital and operation costs calculation

\begin{tabular}{|l|l|r|}
\hline$C e$ & Variable electrical energy cost & $0.055 € / \mathrm{kWh}$ \\
\hline
\end{tabular}




\begin{tabular}{|l|l|r|}
\hline$C F_{C}$ & Capacity factor & $90 \%$ \\
\hline$T h_{a}$ & Total annual hours & 8766 hours \\
\hline$\eta_{c}$ & Compressor efficiency & $70 \%$ \\
\hline$C_{b, C}$ & Base compressor cost & $1,164 € / \mathrm{kW}$ \\
\hline$S_{b, C}$ & Base compressor size & $4,000 \mathrm{~kW}$ \\
\hline$P_{b, C}$ & Baseline operating pressure for compression & $200 \mathrm{bar}$ \\
\hline$S_{b, S}$ & Base tank capacity & $1,252.5 \mathrm{~kg}$ \\
\hline$P_{b, S}$ & Baseline operating pressure for storage & $540 \mathrm{bar}$ \\
\hline
\end{tabular}

Source: [Drennen \& Rosthal, 2007] in € 2015

\section{IV.2.2 Cost of transportation}

The three main capital costs of the CGT go for the truck cab cost $T C_{C a b}$, the truck undercarriage cost $T C_{\text {und }}$ and the tube cost $T C_{\text {tube }}$.

The capital cost related to the purchase of truck components is defined as those for two components the trailer, which includes the undercarriage plus the tube (Equation 13, b), and the cab (Equation 13, a):

$$
\begin{array}{ll}
C C_{C a b}{ }^{t}=n_{T}^{t}\left(T C_{C a b}\right) & a) \\
C C_{T r a}^{t}=n_{T}^{t}\left(T C_{t u b e}+T C_{\text {und }}\right) & \text { b) }
\end{array}
$$

In addition to the capital costs, the fuel cost to perform $n_{w / b}{ }^{t}$ annual roundtrips with $n_{T}{ }^{t}$ truck depend on the roundtrip distance and the unit fuel cost $F_{p}$ in euro/km shown in Equation 14:

$\boldsymbol{F C}=\mathbf{2} * \boldsymbol{n}_{\boldsymbol{T}}^{t} * \boldsymbol{n}_{r . t}{ }^{t} * \boldsymbol{F}_{\boldsymbol{p}} * \boldsymbol{d}_{i j}$

And finally, the annual labour cost $L C$ for $n_{T}{ }^{t}$ truck is calculated by multiplying the numbers of drivers by the driver wage $T C_{d r i v e r}$ as shown in Equation 15:

$L C=n_{T}{ }^{t} * n_{r . t}{ }^{t} * n_{\text {driver }} * T C_{d r i v e r} *\left(\frac{2 . d_{i j}}{S_{a}}+t t_{l / u^{t}}\right)$

Equation 16 shows the total annual cost, while Table 3 lists the definitions and the values of the different parameters used for the calculation:

Table 3: Fixed economic parameters for CG cost calculations 


\begin{tabular}{|l|c|l|}
\hline Cab cost: & $T C_{c a b}$ & $104,740 €$ \\
\hline Average speed & $S a$ & $50 \mathrm{~km} / \mathrm{h}$ \\
\hline
\end{tabular}

Source: [Drennen \& Rosthal, 2007] in € 2015

IEK-STE 2017

$\mathrm{LCOH}_{T} * \boldsymbol{C p}=\left(\mathrm{CRF}_{\mathrm{Tra}}+O \mathrm{M}_{\mathrm{Tra} a}\right) * C C_{T, t r a}{ }^{t}+\left(C R F_{C a b}+O M_{c a b}\right) * C C_{T, c a b}{ }^{t}+F C+L C$

\section{Model calculations and results}

\section{V.1 Mathematical formulation}

The mathematical formulation to identify the optimum combination of CGT giving the minimum cost of transportation for a given total hydrogen demand $C p$ to be transported over a distance $d_{i j}$ can be written as a minimization cost problem shown in Equation 17:

$C C\left(d_{i j}, C p\right)=\min \sum_{t} C_{a n}^{t}$

Where $C^{t}{ }_{a n}$ is the annual cost associated with the CGT operating at the pressure level $t$.

This can be calculated as a product of its total levelized cost LCOTH and the capacity transported by each CGT $C p^{t}$, under the condition that the sum of the capacities transported by the trucks meets the total hydrogen demand $C p$ as shown in Equation 18.

$C C\left(C p^{t}\right)=\min \sum_{t} L C O T H * C p^{t}$

with $C p=\sum_{t} C_{p}^{t}$

The total levelized cost LCOTH can be bring down to the sum of its three components associated to compression (Equation 11), storage (Equation 12) and road transportation (Equation 16). Allowing the minimization problem of Equation 18 to be equivalent of the problem of Equation 19:

$C\left(C p^{t}\right)=\min \sum_{t} C p^{t} *\left[\operatorname{LCOH}_{T}\left(C p^{t}\right)+\chi\left(C p^{t}\right) *\left(\operatorname{LCOH}_{C}\left(C p^{t}\right)+\operatorname{LCOH}_{S}\left(C p^{t}\right)\right)\right]$

with $C p=\sum_{t} C^{t}$

$\chi\left(C p^{t}\right)$ is a function indicating if or if not a compression and storage facility operating at a pressure $P_{t}$ should be implemented. Therefore, it is equal to 0 when there is no capacity transported at this pressure level (i.e. $C p^{t}=0$ ) and equal to 1 otherwise.

The simplification of this problem is done by considering the capacity and the distance, like the cost function, as a discrete variable defined by a variable capacity step $\Delta C p$ and a fixed distance step $\Delta d$ (Equation 19): 
The distance step is independent of the cost optimisation and is chosen constant equal to $1 \mathrm{~km}$ for a distance range reaching $500 \mathrm{~km}$. The capacity step is assumed variable depending on the distance step and ranges from 0.01 TPD to 100 TPD.

In fact the lowest capacities that can be transported by one truck are $720 \mathrm{~kg}$ and $907 \mathrm{~kg}$ respectively corresponding to 250 bar and 350 bar. Therefore, a capacity step $\Delta m$ of $800 \mathrm{~kg}$ is chosen with the maximum loading and unloading time of two hours.

The annual capacity step $\Delta C p$ (Equation 20) is then the product of one CGT capacity step $\Delta m$ and the annual round trip (Equation 3 ) of a CGT of a maximum total loading and unloading time $t t_{l / u^{\max }}$ equal to two hours:

$\left.\Delta \boldsymbol{C p}=\Delta \boldsymbol{m} * \mid \frac{S_{a^{*} A v .}}{2 * \Delta d * p+S_{a^{*} t t_{l / u}{ }^{\max }}}\right\rfloor \quad$ with $p \in \mathbb{N}$

The problem of Equation 18 is equivalent then to the problem shown by Equation 21:

$C(n)=\min _{i_{t} \in[1, n]} \sum_{t} \Delta C p * i^{t} *\left[\operatorname{LCOH}_{T}\left(i^{t}\right)+\chi_{[1, n]}\left(i^{t}\right) *\left(\operatorname{LCOH}_{C}\left(i^{t}\right)+\operatorname{LCOH}_{S}\left(i^{t}\right)\right)\right]$

with $\left\{\begin{array}{l}n=\sum_{t} i^{t} \\ i^{t} \in[0, n]\end{array}\right.$

\section{V.2 Model results}

\section{V.2.1 Minimum cost results}

To see the distribution of the total cost for different distances and capacities, Figure 1 shows the levelized cost of transporting hydrogen for different transported hydrogen capacities below 5 TPD and trip distances ranging from 1 to $500 \mathrm{~km}$. 


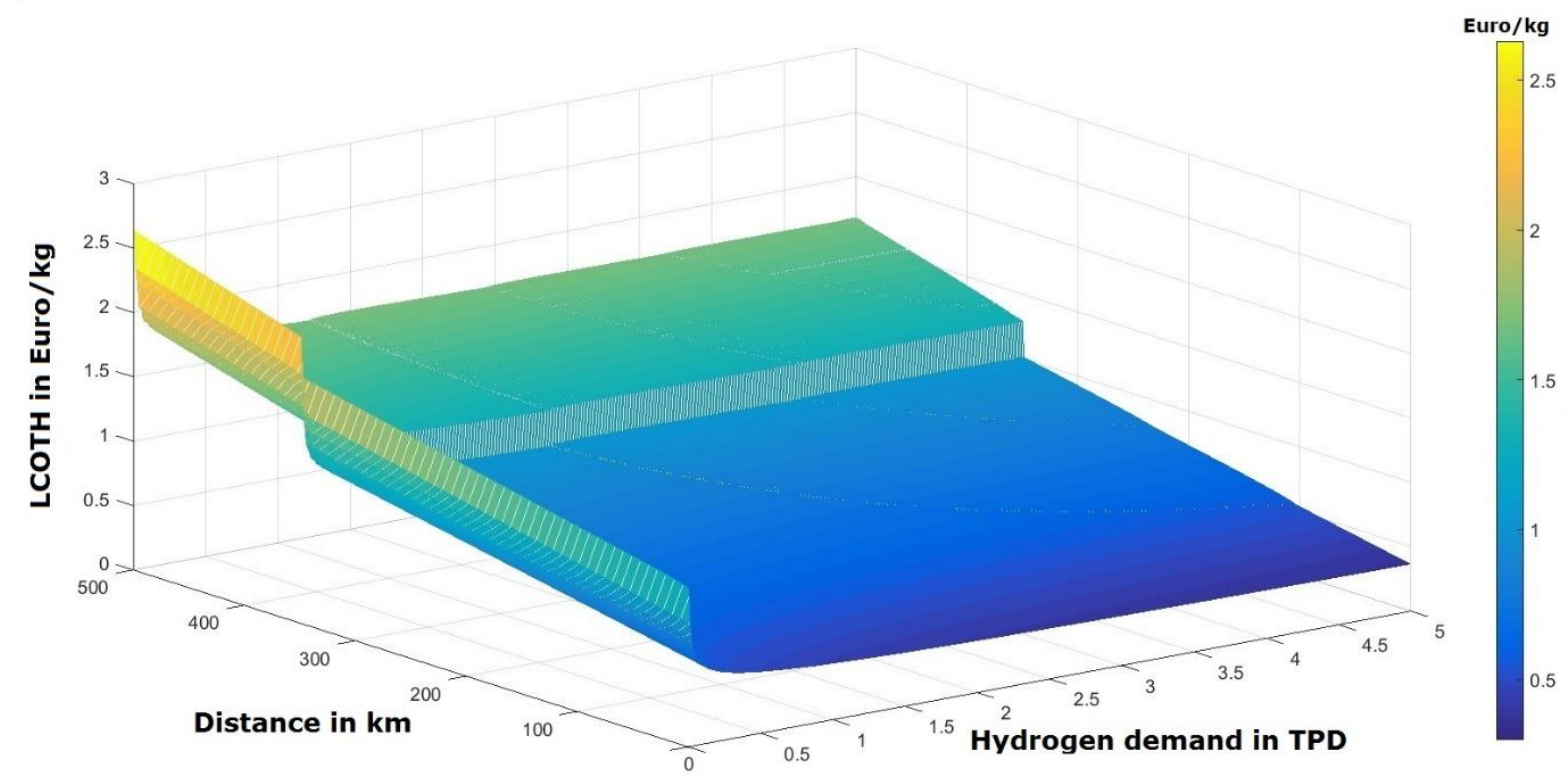

It can be noticed from Figure 1 that the $L C O T H$ is in average continuously changing over capacity and distance; decreasing with the hydrogen transported capacity and increasing with the trip distance.

In fact, the decreasing cost with the hydrogen demand shows the importance of the economy of scale to reduce the total cost of transporting hydrogen. As can be noticed, LCOTH is high below 0.5 TPD as it varies between 2.0 and $2.7 € / \mathrm{kg}$ and decreases when the demand increases. This is due to the fact that at low hydrogen demand the main cost results from installing the different facilities including the storage and compression facilities and the part of capital costs of the different trucks.

The cost increases as well with the distance trip because of the increase of fuel costs and peaks with an additional $0.6 € / \mathrm{kg}$ around $350 \mathrm{~km}$ due to labour costs. In fact the limiting working hours for the driver makes it necessary that two drivers are needed from a certain distance doubling the logistic costs consisting mainly of the driver's wage.

To investigate the impact of the distance and the total hydrogen transported capacity, Figure 2 shows the share of different costs on the total cost of transporting hydrogen; in blue the share of the transportation cost components (capital, fuel and logistic costs) along with the total levelized cost associated with it $\mathrm{LCOH}_{T}$; in orange the share of compression components (capital and compression work cost) and hydrogen storage cost.

These costs are compared for two different values of hydrogen transported capacity counting for 1 TPD at low hydrogen demand and 50 TPD at high hydrogen demand and four trip distances of $20 \mathrm{~km}, 75 \mathrm{~km}, 250 \mathrm{~km}$ and $400 \mathrm{~km}$. 




Figure 2 shows that at low hydrogen demand the cost of compression and storage accounts only for less than $30 \%$ of the total cost regardless of the trip distance. The trip distance impacts the fuel cost that increases over the capital cost when the distance increases.

At high hydrogen demand, the trip distance impacts both costs. In fact, the share of compression and storage on the total cost of transporting hydrogen is decreasing with the distance when transportation costs increase. For instance, it decreased from a share of $70 \%$ at $20 \mathrm{~km}$ to a share of less than $30 \%$ below $250 \mathrm{~km}$ and equally account for the same share of transportation costs at a trip distance of $75 \mathrm{~km}$. 
It can be noticed as well that the general development of transportation cost is different at low and high compression level, at low demand the cost (as shown by $\mathrm{LCOH}_{T}$ ) is mainly constant, except at distances below $75 \mathrm{~km}$ and at $350 \mathrm{~km}$, when the cost for the second driver arise. At high demand it is constantly increasing until it peaks as well around $350 \mathrm{~km}$ as a consequence of the increase of wage cost.

This sudden increase of wage cost impacts on the share of fuel cost as well. Even though fuel cost is more important at $400 \mathrm{~km}$ than at $250 \mathrm{~km}$, its share decreases due to the rise of wage cost.

For compression cost components, the compression work accounts for the main cost at high hydrogen demand and distances round trip below $100 \mathrm{~km}$ due to the low cost of transporting hydrogen $\left(\mathrm{LCOH}_{T}\right)$. This cost decreases with the distance along with capital cost of compression due to the increase of costs related to transportation.

Finally, the average share of capital cost is unchanged between low and high hydrogen demand suggesting that at even high quantity of hydrogen need the cost decreased due to the economy of scale.

\section{V.2.2 Transported capacity using different pressure level}

Figure 3 shows the average share of different CGT used to transport the hydrogen. This is done by taking the mean at each $100 \mathrm{~km}$ to visualize the general distribution of the share of CGT with the distance and the demand. 


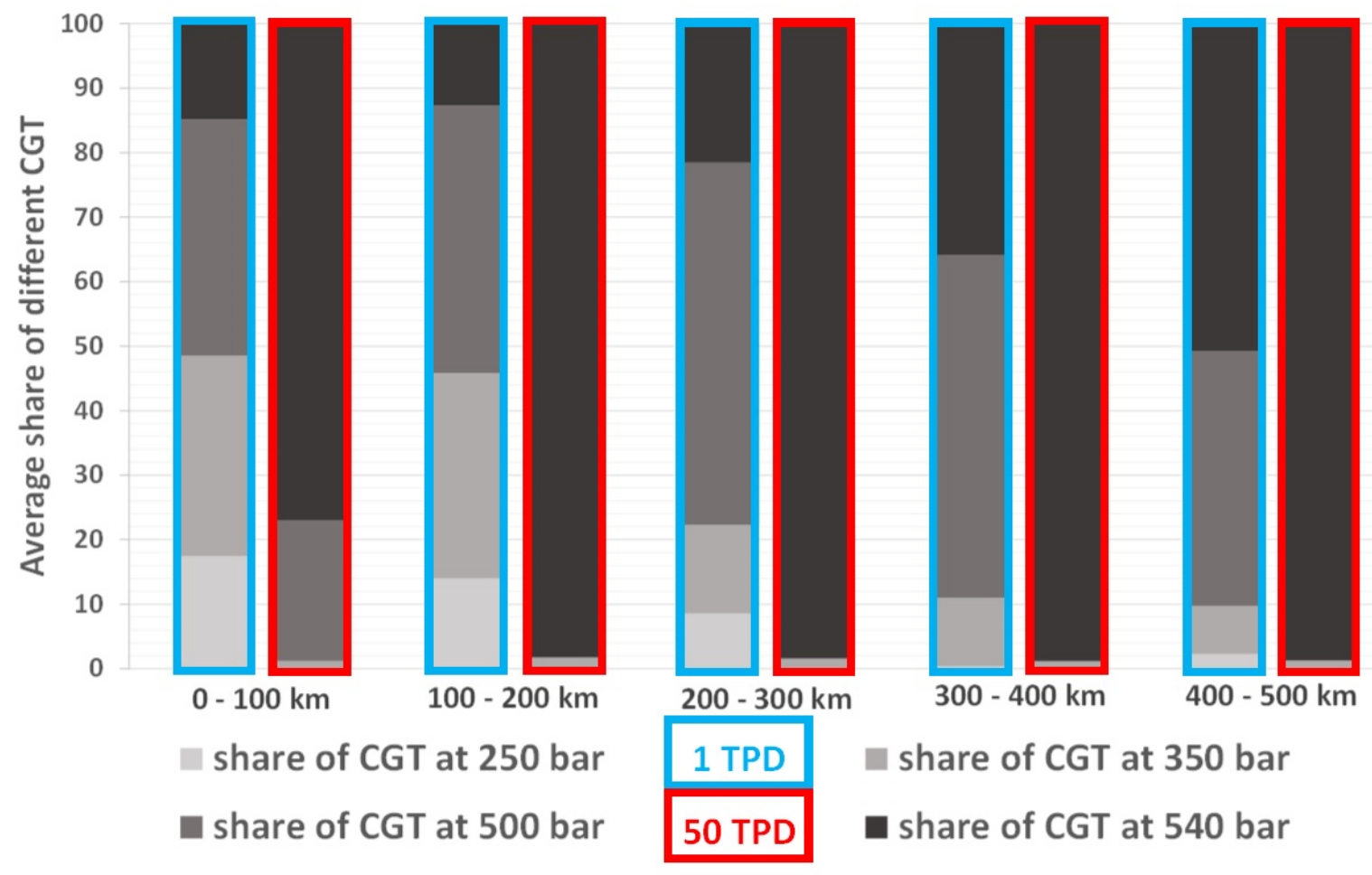

Figure 3 shows that in average and at low hydrogen demand, more CGT at low pressure level are needed at distances below $200 \mathrm{~km}$ as capital cost related to them is lower. When the distance increases, the fuel cost increases as shown in Figure 2. That suggests that less CGT will reduce the cost which apply the use of higher pressure level. As a consequence, the share of CGT at 540 bar is increasing from less than $15 \%$ below $100 \mathrm{~km}$ to the half of the total used CGT between 400 and $500 \mathrm{~km}$ even for a low hydrogen demand.

At high hydrogen demand, on the one hand at low distance below $20 \mathrm{~km}$, the main cost is due to the work of compression, as shown in Figure 2, suggesting the use of low pressure levels. On the other hand, for distances longer $20 \mathrm{~km}$, the transportation cost increases and is equal to the total compression cost at $75 \mathrm{~km}$ suggesting the use of higher CGT to meet the higher hydrogen demand at lower cost. Taking that into account, below $100 \mathrm{~km}$ a compromise between lower pressure level below $20 \mathrm{~km}$ and higher between 20 and $100 \mathrm{~km}$ has to be found. On an average distance between 0 and $100 \mathrm{~km}$ this is reflected in a use of $22 \%$ CGT at 500 bar and more than $75 \%$ of GGT at 540 bar.

At high demand and for more than $100 \mathrm{~km}$ trip distance, the hydrogen is transported using almost only CGT at 540 bar reflected by a share of $98-99 \%$ when the rest goes for CGT at lower pressure 
level to meet the fluctuation of hydrogen demand. In fact, lower pressure is needed to transport the additional quantity missing at to lower cost.

\section{V.3 Results comparison and future prospects}

First, some results obtained were compared to the literature, then a review of the cost of different transportation modes was performed in order to give future prospects to the current work.

\section{V.3.1 Results comparison to the literature}

For the optimal CGT, [Reddi et al., 2018] evaluated the impact of three CGT configurations (of 600,690 and $790 \mathrm{~kg}$ capacity) on the transportation and refuelling cost. The simulation was performed in order to deliver hydrogen over different transportation distances of 100, 200 and 300 miles and to hydrogen refuelling stations of various capacities of 250,500 and $750 \mathrm{~kg} / \mathrm{day}$ corresponding to low hydrogen demand (around $200 \mathrm{~kg} /$ day), medium hydrogen demand (around $400 \mathrm{~kg} /$ day) and high hydrogen demand ( around $1000 \mathrm{~kg} / \mathrm{day}$ ).

Table 4 compare the results at low and high hydrogen demand for two distances of $161 \mathrm{~km}$ and $483 \mathrm{~km}$. The results in red correspond to the literature results [Reddi et al., 2018] and show the CGT that gives the minimum cost. The results in black, correspond to the study results and show the CGT that is used the most to deliver the hydrogen demand.

Table 4: CGT configuration giving the minimum transport cost at 161 and $483 \mathrm{~km}$ for low and high demand

\begin{tabular}{|l|l|l|l|}
\hline \multicolumn{2}{|c|}{ Low hydrogen demand } & \multicolumn{2}{|c|}{ High hydrogen demand } \\
\hline Refuelling station capacity of $250 \mathrm{~kg} /$ day & \multicolumn{2}{|c|}{ Hefuelling station capacity of 750kg/day* } \\
\hline Distance of $161 \mathrm{~km}$ & Distance of $483 \mathrm{~km}$ & Distance of $161 \mathrm{~km}$ & Distance of $483 \mathrm{~km}$ \\
\hline CGT at $600 \mathrm{~kg} *$ & CGT at $790 \mathrm{~kg} *$ & CGT at $790 \mathrm{~kg} *$ \\
\hline
\end{tabular}

Source: [Reddi et al., 2018] *

For each configuration, the tube trailer cost contribution was find out to increase as well with the distance and to decrease with the hydrogen demand. At high hydrogen demand, the results correspond to the conclusion of the study performed, as the CGT with higher capacity is find out to deliver the hydrogen at the minimum cost independently from the distance of transport.

At low hydrogen demand, both studies show the impact of the distance in the CGT used. In fact, CGT with lower capacities are used at $161 \mathrm{~km}$ distance trip, when the ones at higher capacities are used to transport hydrogen over $483 \mathrm{~km}$ distance trip. 
To visualize the impact of the distance on the different costs, operation and maintenance costs for both compression and transport were compared to the capital costs. For that, the fuel cost, the wage cost and transport operations cost were summed under transport OM; and compression work cost and operations cost under compression OM. The results were compared to the work of [Yang \& Ogden, 2007] that gives the four cost in addition to storage cost for a transported hydrogen capacity of 15 TPD over two roundtrip distances of 50 and $300 \mathrm{~km}$.

Table 5 show the different literature costs share in red along with the study results for storage cost, transport capital cost (transport CC), transport operations and maintenance cost (transport OM), compression capital cost (compression CC), and compression operations and maintenance cost (compression OM),

Table 5: Share of different cost components for a hydrogen demand of 15 TPD over a distance roundtrip of 50 and $300 \mathrm{~km}$

\begin{tabular}{|c|c|c|}
\hline Distance & $50 \mathrm{~km}$ & $300 \mathrm{~km}$ \\
\hline Storage & & \\
\hline Transport CC & & \\
\hline
\end{tabular}



Source: [Yang \& Ogden, 2007] * in € 2015

For both sources, the main cost comes from the transport OM, followed by the transport CC. The order of magnitude in the other hand, varies for both studies due to different investment cost of the tube for transport CC. In fact, [Yang \& Ogden, 2007] investigates only one type of CGT transporting a capacity of $300 \mathrm{~kg}$, when the study investigated a combination of four different CGT. This difference impacts as well the capacity transported by a singles CGT and therefore the fuel cost.

The share of storage cost varies as well because of the different tanks cost that were used to store hydrogen.

Finally, the difference in Compression CC and Compression OM is due to the use of higher pressure to transport hydrogen which impacts the energy use. For instance [Yang \& Ogden, 2007] uses a compressor with energy use between 0.7 and $1 \mathrm{kWh} / \mathrm{kg}$, when the study performed has a compression work that varies between 3.1 and $3.7 \mathrm{kWh} / \mathrm{kg}$.

\section{V.3.2 Future prospects}

As concluded in the results section, the use of CGT is mainly effective between distances ranging between $75 \mathrm{~km}$ and $350 \mathrm{~km}$ and a low to average demand over one TPD. This suggests the use of 
other forms of transporting hydrogen and other infrastructure system than the road transportation network.

A literature review gives an overview of other modes of transportation and which cost could be reduced and at which range.

For instance, a study to support the transition towards a low-carbon transport system in UK was done taking into account two discounts rate of $3.5 \%$ and $10 \%$ and two modes of transporting hydrogen including pipeline system and compressed gas [Moreno-Benito et al., 2017]. For the case of $10 \%$ discount rate, 10 scenarios were analysed that depend on different international hydrogen import and different pipeline capital cost and potential connexion. Thus, the average cost was taken excluding the costs related to the imports and carbon emissions to allow the comparison between the transportation cost components. The results showed in one hand, that road transportation operations and maintenance costs were more important than pipelines ones and accounted for $13.95 \%$ instead of only $0.32 \%$ of total transportation cost. In the other hand, the capital cost to construct pipeline system was three times more important. This observation suggests, that at very high hydrogen demand the pipelines capital cost could be reduced due to the economy of scale, allowing as well to avoid the total operations and maintenance of compressed gas (including the wage cost) that represent the main cost share.

The general study performed by [Yang \& Ogden, 2007] confirms the comparison between the two modes of transportation at larger scale, as it compared the costs at different transport distance and hydrogen demand. For instance, the pipeline system was found out to be cost effective at 100 TPD and $300 \mathrm{~km}$, when CGT was cost effective at $50 \mathrm{~km}$ and 15 TPD. The study suggests as well, that liquid hydrogen could be a good transition option between the two. For instance, at $300 \mathrm{~km}$ and 15 TPD, even with high share of liquefaction work that counts for $29 \%$, the CGT total operation and maintenance cost were reduced from a share of $57 \%$ to only $8 \%$ due to the use of less cost wages and trucks.

The use of liquefied hydrogen could be as well cost effective at high energy demand in long term perspectives [Cardella et al., 2017]. In fact, the total liquefaction cost could be reduced by nearly 0.66 euro/ kg when comparing current 50 TPD installed capacity with optimized large-scale liquefiers up to 150 TPD liquefaction capacity.

At low hydrogen demand, another alternative should be investigated as a tread off should be found between higher operations and maintenance cost when using CGT, and higher investment cost in case of using liquid hydrogen or pipelines [Demir \& Dincer, 2018]. For instance, at 4.37 TPD, liquid hydrogen transport showed a lower operations and maintenance cost share, but higher investment cost increasing the total costs by $280 \%$. When transportation via pipeline system showed a same total cost as CGT at 4.36 TPD but for higher hydrogen demand reaching 79.95 TPD. 


\section{Conclusion}

This study offers a complementary analysis to the existing literature concerning optimizing the hydrogen transportation supply chain. Further improvement on transporting hydrogen using compressed gas trucks by assessing four pressure levels at different hydrogen demand ranges and distance transportation trips were investigated.

The pressure levels ranged from 250 to 540 bar and correspond to the current and prospects of the hydrogen market. The transported hydrogen is meant to be used in mobility sector as an alternative fuel for FCEVs. Thus, the hydrogen demand was fixed as an input parameter reaching a maximum capacity of 100 TPD for regional use. Hydrogen was then compressed, stored and transported using gas trucks over a distance ranging between $1 \mathrm{~km}$ and $500 \mathrm{~km}$ for regional use.

First, compression cost is derived from the work of a five-stage compressor based on its adiabatic work and total efficiency that includes adiabatic and electrical ones. Then, both capital costs of storage and compression were calculated using a sizing factor based on the pressure levels of 200 and 540 bar. Finally, capital cost of truck components, fuel and wage costs were added, and the total cost were minimized by optimizing the capacities transported by each truck.

The analysis showed that the total cost of transporting hydrogen decreases with the transported capacity. In fact, the total levelized cost of transporting hydrogen is high (between 2 and $2.7 € / \mathrm{kg}$ ) below 0.5 ton per day (TPD) due to higher investment costs on the truck components, storage and compression facilities and decreases by more than $1 € / \mathrm{kg}$ at transported capacity below one TPD due to the economy of scale.

In parallel, the overall cost increases as well with the trip distance because of the fuel cost, and peaks with an additional $0.6 € / \mathrm{kg}$ around $350 \mathrm{~km}$ due to logistic costs. In fact, the limiting working hours require the deployment of two drivers simultaneously from a distance trip above $350 \mathrm{~km}$.

The analysis investigates the different pressure level distribution as a function of distance trip and hydrogen demand. The results showe that the share of compressed gas trucks operating at the highest-pressure level of 540 bar increases with the distance and hydrogen demand. In contrast, the share of trucks transporting hydrogen at lower pressure level of 250 and 350 bar is higher at trip distance below $200 \mathrm{~km}$.

Finally, the use of CGT is found out to be mainly effective between a distance ranging between 75 and $350 \mathrm{~km}$ and a transported capacity between 1 and 50 TPD, where the cost components 
are continuously involving without any peaks. Which suggests the use of other forms of transporting hydrogen and other infrastructure system than the road transportation network.

For instance, at transported capacities below one TPD, the high cost arise mainly in capital cost. Therefore, the use of more standard tubes as those used for transporting hydrogen could reduce the costs. One possibility would be to bind the hydrogen to a liquid organic hydrogen carrier (LOHC).

With a higher transported capacity and at trip distance below $75 \mathrm{~km}$, the main cost derive from the work needed to compress hydrogen at high pressures. The use of a pipeline transportation system could offer a good compromise between less compression work and larger capacities of transported hydrogen.

Finally, at a larger transported capacity over 50 TPD and at transportation distance over $350 \mathrm{~km}$, the main cost arise in the CGT logistic cost that can be reduced using less trucks; that can be done by changing the transportation infrastructure to the pipeline system or using a liquefied hydrogen trucks to increase the annual capacity transported.

\section{References}

ALMANSOORI, A. \& SHAH, N. (2006) Design and operation of a future hydrogen supply chain: snapshot model. Chemical Engineering Research and Design, 84:6, 423-438.

ANDRÉ, J., AURAY, S., DE WOLF, D., MEMMAH, M.-M. \& SIMONNET, A. (2014) Time development of new hydrogen transmission pipeline networks for France. International Journal of Hydrogen Energy, 39:20, 10323-10337.

BAUFUMÉ, S., GRÜGER, F., GRUBE, T., KRIEG, D., LINSSEN, J., WEBER, M., HAKE, J.-F. \& STOLTEN, D. (2013) GIS-based scenario calculations for a nationwide German hydrogen pipeline infrastructure. International Journal of Hydrogen Energy, 38:10, 3813-3829.

BELLOTTI, D., RIVAROLO, M., MAGISTRI, L. \& MASSARDO, A. F. (2015) Thermoeconomic comparison of hydrogen and hydro-methane produced from hydroelectric energy for land transportation. International Journal of Hydrogen Energy, 40:6, 2433-2444.

CARDELLA, U., DECKER, L. \& KLEIN, H. (2017) Roadmap to economically viable hydrogen liquefaction. International Journal of Hydrogen Energy, 42:19, 1332913338.

COMMISSION, E. (2015) EU transport in figures.

COMPOSITES, H. (2006) An advanced pipeline to supply energy gases over long distances at competitive costs. mobile pipeline

DAYHIM, M., JAFARI, M. A. \& MAZUREK, M. (2014) Planning sustainable hydrogen supply chain infrastructure with uncertain demand. International Journal of Hydrogen Energy, 39:13, 6789-6801. 
DEMIR, M. E. \& DINCER, I. (2017) Cost assessment and evaluation of various hydrogen delivery scenarios. International Journal of Hydrogen Energy.

DEMIR, M. E. \& DINCER, I. (2018) Cost assessment and evaluation of various hydrogen delivery scenarios. International Journal of Hydrogen Energy, 43:22, 10420-10430.

DRENNEN, T. E. \& ROSTHAL, J. E. (2007) Pathways to a hydrogen future, Elsevier Science.

EOLIENNE, F. E. (2017) La transition énergétique. http://fee.asso.fr/politique-deleolien/la-transition-energetique/.

GERBONI, R. (2016) 11 - Introduction to hydrogen transportation A2 - Gupta, Ram B. In BASILE, A. \& VEZIROĞLU, T. N. (Eds.) Compendium of Hydrogen Energy.283299, Woodhead Publishing.

GOUVERNEMT (August 2017) Plan Climat. http://www.gouvernement.fr/action/planclimat.

HE, C., SUN, H., XU, Y. \& LV, S. (2017) Hydrogen refueling station siting of expressway based on the optimization of hydrogen life cycle cost. International Journal of Hydrogen Energy, 42:26, 16313-16324.

HEXAGON, L. (2013) Development of High Pressure Hydrogen Storage Tank for Storage and Gaseous Truck Delivery. $\mathrm{H} 2$ program.

JENSEN, J. O., VESTBØ, A. P., LI, Q. \& BJERRUM, N. J. (2007) The energy efficiency of onboard hydrogen storage. Journal of Alloys and Compounds, 446447:Supplement C, 723-728.

KIM, J.-G. \& KUBY, M. (2012) The deviation-flow refueling location model for optimizing a network of refueling stations. International Journal of Hydrogen Energy, 37:6, 5406-5420.

LAHNAOUI, A., WULF, C., HEINRICHS, H. \& DALMAZZONE, D. (2017) Optimizing hydrogen transportation system for mobility via compressed hydrogen trucks. International Journal of Hydrogen Energy:In process.

MARCOULAKI, E. C., PAPAZOGLOU, I. A. \& PIXOPOULOU, N. (2012) Integrated framework for the design of pipeline systems using stochastic optimisation and GIS tools. Chemical Engineering Research and Design, 90:12, 2209-2222.

MCCARTY, R. D., HORD, J. \& RODER, H. (1981) Selected properties of hydrogen (engineering design data). Final report. National Engineering Lab.(NBS), Boulder, CO (USA).

OPSD (2018) Renewable power plants DE. https://data.open-power-systemdata.org/renewable power plants/. 29th March 2018.

REDDI, K., ELGOWAINY, A., RUSTAGI, N. \& GUPTA, E. (2018) Techno-economic analysis of conventional and advanced high-pressure tube trailer configurations for compressed hydrogen gas transportation and refueling. International Journal of Hydrogen Energy, 43:9, 4428-4438.

SHORT, W., PACKEY, D. J., HOLT, T. \& NATIONAL RENEWABLE ENERGY, L. (2005) A manual for the economic evaluation of energy efficiency and renewable energy technologies. Honolulu, Hawaii, University Press of the Pacific.

SIMBECK, D. \& CHANG, E. (2002a) Hydrogen supply: cost estimate for hydrogen pathways-scoping analysis. NREL, Bolden, Colorado. 
SIMBECK, D. \& CHANG, E. (2002b) Hydrogen supply: cost estimate for hydrogen pathways-scoping analysis. National Renewable Energy Laboratory, 71.

STEWARD, D., RAMSDEN, T., ZUBOY, J. \& NATIONAL RENEWABLE ENERGY, L. (2008) H2A Production Model, Version 2 user guide.

STOJIĆ, D. L., MARČETA, M. P., SOVILJ, S. P. \& MILJANIĆ, Š. S. (2003) Hydrogen generation from water electrolysis-possibilities of energy saving. Journal of Power Sources, 118:1, 315-319.

SUN, H., HE, C., WANG, H., ZHANG, Y., LV, S. \& XU, Y. (2017) Hydrogen station siting optimization based on multi-source hydrogen supply and life cycle cost. International Journal of Hydrogen Energy, 42:38, 23952-23965.

TAMHANKAR, S. (2014) Terminal Operations for Tube Trailer and Liquid Tanker Filling: Status, Challenges and R\&D Needs. DOE Hydrogen Transmission and Distribution Workshop, Golden, CO, Feb 25-26, 2014.

YANG, C. \& OGDEN, J. (2007) Determining the lowest-cost hydrogen delivery mode. International Journal of Hydrogen Energy, 32:2, 268-286.

ZERHUSEN (2013) IMPACT OF HIGH CAPACITY CGH2-TRAILERS. DeliverHy partners, DeliverHy partners. 